\title{
A novel approach to select differential pathways associated with hypertrophic cardiomyopathy based on gene co-expression analysis
}

\author{
XIAO-MIN CHEN $^{1 *}$, MING-JUN FENG $^{1 *}$, CAI-JIE SHEN ${ }^{2}$, BIN HE $^{1}$, \\ XIAN-FENG DU ${ }^{1}$, YI-BO YU ${ }^{1}$, JING LIU ${ }^{1}$ and HUI-MIN CHU ${ }^{1}$ \\ ${ }^{1}$ Department of Cardiology, Ningbo First Hospital; ${ }^{2}$ Department of Cardiology, \\ Ningbo Seventh Hospital, Ningbo, Zhejiang 315000, P.R. China
}

Received April 19, 2016; Accepted March 20, 2017

DOI: $10.3892 / \mathrm{mmr} .2017 .6667$

\begin{abstract}
The present study was designed to develop a novel method for identifying significant pathways associated with human hypertrophic cardiomyopathy (HCM), based on gene co-expression analysis. The microarray dataset associated with HCM (E-GEOD-36961) was obtained from the European Molecular Biology Laboratory-European Bioinformatics Institute database. Informative pathways were selected based on the Reactome pathway database and screening treatments. An empirical Bayes method was utilized to construct co-expression networks for informative pathways, and a weight value was assigned to each pathway. Differential pathways were extracted based on weight threshold, which was calculated using a random model. In order to assess whether the co-expression method was feasible, it was compared with traditional pathway enrichment analysis of differentially expressed genes, which were identified using the significance analysis of microarrays package. A total of 1,074 informative pathways were screened out for subsequent investigations and their weight values were also obtained. According to the threshold of weight value of $0.01057,447$ differential pathways, including folding of actin by chaperonin containing T-complex protein 1 (CCT)/T-complex protein 1 ring complex (TRiC), purine ribonucleoside monophosphate biosynthesis and ubiquinol biosynthesis, were obtained. Compared with traditional pathway enrichment analysis, the number of pathways obtained from the co-expression approach was increased. The results of the present study demonstrated that this method may
\end{abstract}

Correspondence to: Dr Hui-Min Chu, Department of Cardiology, Ningbo First Hospital, 59 Liuting Street, Ningbo, Zhejiang 315000, P.R. China

E-mail: chuhuiminhang@163.com

*Contributed equally

Key words: hypertrophic cardiomyopathy, differentially expressed genes, differential pathways, co-expression network be useful to predict marker pathways for HCM. The pathways of folding of actin by CCT/TRiC and purine ribonucleoside monophosphate biosynthesis may provide evidence of the underlying molecular mechanisms of HCM, and offer novel therapeutic directions for HCM.

\section{Introduction}

Hypertrophic cardiomyopathy (HCM) is a complex and relatively common genetic heart disease, which is characterized by unexplained asymmetric or symmetric cardiac hypertrophy, interstitial fibrosis and cardiomyocyte disarray (1). HCM is a prevalent disease that affects $0.2 \%$ of the global population (2). Patients with HCM may suffer from early sudden cardiac death which is most common in individuals $<35$ years of age $(3,4)$. Therefore, it is necessary to identify effective therapeutic strategies and investigate the etiology of HCM.

The development of large scale microarray analyses has led to research at the gene level, which may be a powerful and informative means of investigating the mechanism of a disease (5). HCM is predominantly induced by mutations in genes encoding sarcomere proteins, including myosin heavy chain 7, myosin light chain 2 and myosin binding protein $C$, cardiac $(6,7)$. HCM-associated genes are typically selected by analyzing differentially expressed genes (DEGs). However, a previous study identified inconsistencies between numerous gene signatures across different studies of the same disease (8). A potentially more effective method is to use pathway analysis in order to evaluate disease-associated biomarkers.

Pathway analysis has been widely used in previous studies (9-11) and is becoming the primary method of obtaining a deep insight into biological processes (12). The identification of active pathways that differ between two conditions is of increased utility compared with a list of DEGs (10). However, existing methods primarily utilize pathway repositories, including the Kyoto Encyclopedia of Genes and Genomes (13), to evaluate whether a pathway is significant by assigning a P-value to the pathway, an approach which focuses on the static regulation between genes $(14,15)$. Analysis of dynamic regulation or network rewiring may reveal important information which may not be identified in static conditions (16). 
Interactions between molecules may alter in different tissues and these alterations are associated with disease progression. Therefore, novel pathway-based biomarkers may be identified by studying dynamic regulation and network rewiring among molecules, in contrast with investigations which only examine differential expression.

In the present study, a novel method was developed to identify pathway-based biomarkers in HCM, by investigating interactions between molecules associated with pathogenesis through a co-expression network strategy. HCM-associated microarray expression data were downloaded from the European Molecular Biology Laboratory-European Bioinformatics Institute (EMBL-EBI) database. The co-expression network was constructed using an empirical Bayes (EB) approach following assignment of a weight value for each pathway. A random model was constructed to define the thresholds to select differential pathways. Pathway enrichment analysis of DEGs, based on the Database for Annotation, Visualization and Integrated Discovery (DAVID) test, was implemented to verify the feasibility of the novel method.

\section{Materials and methods}

Data collection and preprocessing. The HCM-associated microarray expression data, under accession number E-GEOD-36961, were downloaded from the EMBL-EBI database (http://www.ebi.ac.uk/arrayexpress/). In gene expression profile E-GEOD-36961, there were 39 healthy subjects and 106 HCM samples. In order to assess the quality of the data, standard preprocessing of microarray probe data was performed. This preprocessing included the following successive steps: Background correction using robust multiarray analysis (17); normalization using the quartile function to eliminate the influence of nonspecific hybridization (18); perfect match correction using the MAS5.0 algorithm (19); and calculation of expression values from probe intensities. The expression value for every gene was acquired, including 37,846 genes from 145 samples (39 healthy and $106 \mathrm{HCM}$ ).

Pathway data. Reactome is a database which includes numerous pathways in Homo sapiens and a number of reference species, offering an infrastructure for computation across the biological reaction network (20). Human biological pathways were downloaded from the Reactome pathway database (reactome.org). A total of 1,675 pathways were obtained. In order to ensure the validity of the pathways, pathways in which the gene number was $\leq 2$ were discarded. Finally, a total of 1,639 pathways were determined as background pathways.

Construction of a co-expression network using an EB approach. Numerous methods have been demonstrated for co-expression analysis to identify co-expression gene pairs, which include EB (21), Arabidopsis Co-expression Tool (22) and WGCNA (23). In the present study, an EB framework was implemented, which offered a false discovery rate (FDR)-controlled list of the gene pairs of interest (21).

The mean gene number $(\mathrm{N})$ for the 1,639 background pathways was computed based on $\mathrm{N}=$ overall gene number for background pathways $(73,099) /$ the number of background pathways $(1,639)$; therefore, $\mathrm{N}=44.6$. In order to facilitate the analysis, the mean gene number was subsequently defined as $\mathrm{N}=44$.

The gene number of each pathway was termed A, and the number of intersections between each pathway and the gene expression profile was termed B. The informative pathways used in the present study were screened out according to the following conditions: $\mathrm{B}>5$; and the ratio $\mathrm{B}: \mathrm{A}>0.9$. Therefore, $\mathrm{a}$ total of 1,074 informative pathways were selected for further investigation.

Subsequently, the EB approach developed by Dawson and Kendziorski (24) was used to identify co-expression gene pairs among the genes in each informative pathway and construct a co-expression network. The number of possible gene pairs of each informative pathway was termed $\mathrm{C}[\mathrm{C}=\mathrm{A} \times(\mathrm{A}-1) / 2]$. In the $\mathrm{EB}$ method, the identification of co-expression gene pairs was implemented based on the following steps: Three inputs of matrix X; the conditions array; and the pattern object required. The expression levels in an $m \times n$ matrix of $\mathrm{X}$, where $m=$ the number of genes under any informative pathway and $n=$ the total number of representative pathways, were normalized. Subsequently, the members of the conditions array with length $n$ took values in $1,2, \ldots . . K$, where $K$ indicated the number of conditions. Based on matrix $\mathrm{X}$ and the conditions array, intra-group correlations for all gene pairs were computed, and $M$ matrix of correlations was obtained. The Mclust algorithm (25) was used to initialize the hyper-parameters via the initializeHP function of the EBcoexpress package (26), to discover the component normal mixture model which best fitted the correlations of $M$. The crit.fun function of EBcoexpress package (26), was used to define a threshold by controlling the posterior probabilities of co-expression to extract particular co-expression gene pairs. Gene pairs with FDR $\leq 0.05$ were chosen to construct the co-expression network. The number of interactions in one pathway co-expression network was termed $\mathrm{D}$, and $\mathrm{D} / \mathrm{C}$ was recorded as the weight value of the pathway.

Identification of differential pathways. In an attempt to identify differential pathways between HCM and normal samples, a random model consisting of $\mathrm{G}$ genes was constructed. $\mathrm{G}$ genes were randomly selected from gene expression profiles and the weight values for each pathway were calculated using the EB approach. This step was repeated 10,000 times and the weight values were listed in descending order. The FDR for the 100th weight value was set at 0.01 (weight value $=0.01057$ ). The pathways with a weight value $>0.01057$ were considered to be differential pathways.

Identification of DEGs. As previously demonstrated, differential gene expression levels are associated with disease severity. In the current study, the identification of DEGs between HCM and normal samples was performed using the significance analysis of microarrays (SAM) package. The samr function of the SAM package was used to extract the genes which exhibited statistically significant differential expression. Each gene was assigned a score according to the difference in gene expression compared with the standard deviation of repeated measurements for this gene. Genes with scores above the threshold were considered to be potentially significant. The percentage of falsely significant genes relative to the significant genes was defined as the FDR. In order to increase the 
Table I. Top 20 differential pathways identified by the empirical Bayesian analysis.

\begin{tabular}{|c|c|c|}
\hline Row & Weight values & Differential pathways \\
\hline 1 & 0.88889 & Folding of actin by CCT/TRiC \\
\hline 2 & 0.75556 & Purine ribonucleoside monophosphate biosynthesis \\
\hline 3 & 0.42857 & Ubiquinol biosynthesis \\
\hline 4 & 0.33333 & Synthesis of 5-eicosatetraenoic acids \\
\hline 5 & 0.31000 & Cooperation of prefoldin and TRiC/CCT in actin and tubulin folding \\
\hline 6 & 0.30072 & Prefoldin mediated transfer of substrate to CCT/TRiC \\
\hline 7 & 0.28571 & Defective holocarboxylase synthetase causes multiple carboxylase deficiency \\
\hline 8 & 0.28571 & Defects in biotin metabolism \\
\hline 9 & 0.28571 & Mitochondrial fatty acid beta-oxidation \\
\hline 10 & 0.27941 & Branched-chain amino acid catabolism \\
\hline 11 & 0.25974 & Cytosolic tRNA aminoacylation \\
\hline 12 & 0.22807 & Citric acid cycle \\
\hline 13 & 0.22222 & Role of Abl in Robo-Slit signaling \\
\hline 14 & 0.20000 & Uptake and function of diphtheria toxin \\
\hline 15 & 0.19044 & Nonsense mediated decay independent of the exon junction complex \\
\hline 16 & 0.17778 & Zinc influx into cells by the SLC39 gene family \\
\hline 17 & 0.17191 & Eukaryotic translation termination \\
\hline 18 & 0.17177 & GTP hydrolysis and joining of the $60 \mathrm{~S}$ ribosomal subunit \\
\hline 19 & 0.17114 & Cap-dependent translation initiation \\
\hline 20 & 0.17114 & Eukaryotic translation initiation \\
\hline
\end{tabular}

CCT, T-complex protein; TRiC, T-complex protein 1 ring complex.

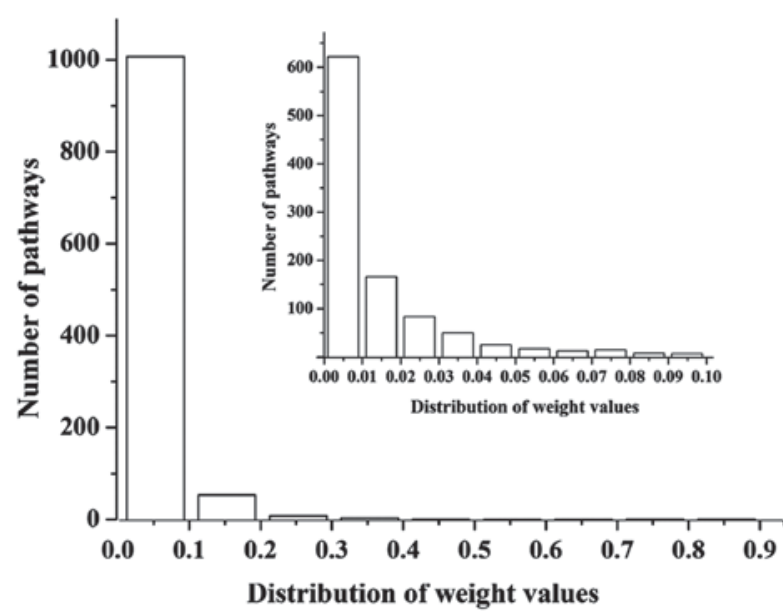

Figure 1. Distribution of weight values of each informative pathway.

stringency for differential gene expression, the delta value was determined using the function SAMR.compute.delta.table. DEGs in HCM were identified for further analysis with the delta threshold value of 7.48 .

Pathway analyses for DEGs. In order to further investigate the biological functions of DEGs, Reactome pathway enrichment analysis was conducted through DAVID (27) by means of the Expression Analysis Systematic Explorer (EASE) test (28). The EASE score was applied to identify the significant categories. In the present study, the pathways with $\mathrm{P}<0.05$ were considered to be significant pathways.

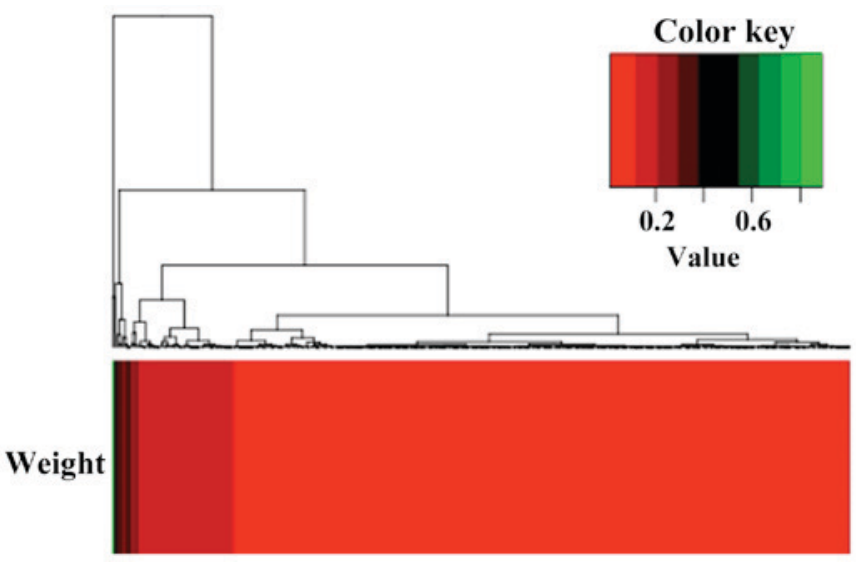

Figure 2. Heat map between differential pathways and their weight values.

\section{Results}

Identification of differential pathways. Following pre-processing, a total of 37,846 genes were obtained and used for subsequent analysis. A total of 1,074 informative pathways were identified, based on the Reactome pathway database and filtration treatment. For each informative pathway, a weight value was calculated based on the EB co-expression network. The distribution of weight values of the pathways are presented in Fig. 1; it was observed that the majority of informative pathways exhibited weight values between 0 and 0.04 , particularly between 0 and 0.01 . An 


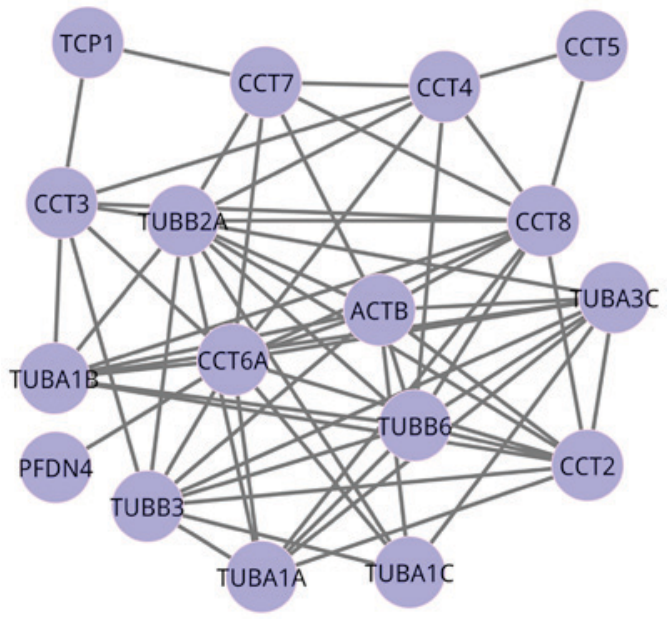

Figure 3. Co-expression network for genes in the differential pathway cooperation of prefoldin and T-complex protein 1 ring complex/T-complex protein 1 in actin and tubulin folding. Nodes, genes; edges, interactions.

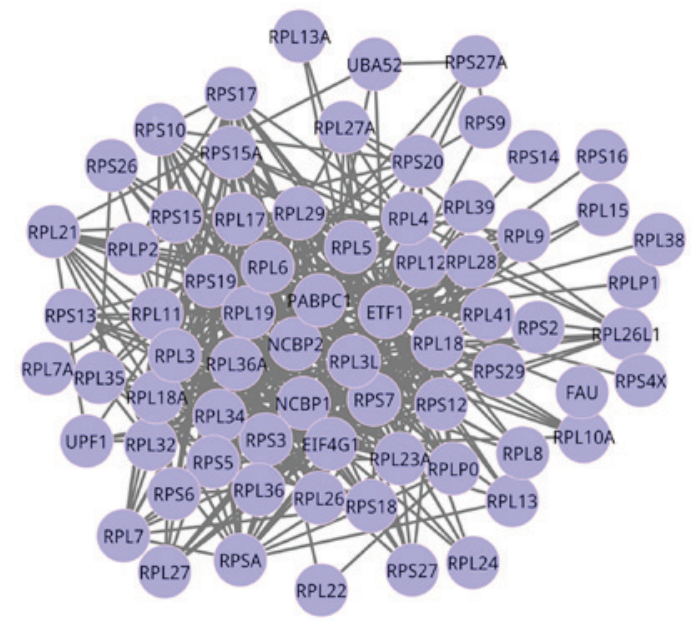

Figure 4. Co-expression network for genes in the differential pathway nonsense mediated decay independent of the exon junction complex. Nodes, genes; edges, interactions. increased weight value indicates a more significant pathway. Therefore, the threshold criteria for differential pathways was defined. In the present study, the threshold for weight values was calculated to be 0.01057 , and 447 differential pathways were selected according to the threshold. The heat-map between differential pathways and the corresponding weight values is presented in Fig. 2. The top 20 differential pathways are presented in Table I. Among these, the top 5 differential pathways were: Folding of actin by chaperonin containing T-complex protein 1 (CCT)/T-complex protein 1 ring complex (TRiC) (weight value=0.88889); purine ribonucleoside monophosphate biosynthesis (weight value $=0.75556$ ); ubiquinol biosynthesis (weight value $=0.42857$ ); synthesis of 5-eicosatetraenoic acids (weight value $=0.33333$ ); and cooperation of prefoldin and TRiC/CCT in actin and tubulin folding (weight value $=0.31000$ ). In order to further elucidate each pathway, networks were constructed using the EB method. As the gene count varied between differential pathways, and too few genes are unable to form a network, differential pathways with an increased number of genes were chosen, including cooperation of prefoldin and $\mathrm{TRiC} / \mathrm{CCT}$ in actin and tubulin folding (gene number=25), and nonsense mediated decay (NMD) independent of the exon junction complex (EJC) (gene number $=88$ ). The co-expression networks of the pathways of cooperation of prefoldin and TRiC/CCT in actin and tubulin folding, and NMD independent of the EJC, are exhibited in Figs. 3 and 4, respectively. For the network of cooperation of prefoldin and TRiC/CCT in actin and tubulin folding, there were 17 genes, while there were 72 genes in the network of NMD independent of the EJC.

Pathway analyses for DEGs using traditional DAVID software. Based on the delta cut-off value of 7.48, a total of 344 DEGs were identified. Pathway analyses indicated that there were only 2 significant pathways based with $\mathrm{P}<0.05$, including signaling in immune system and hemostasis (data not shown).

Comparison of EB and DAVID. In order to assess whether the pathway co-expression network method was feasible, the method developed in the present study was compared with traditional DAVID software. It was observed that the differential pathway of hemostasis was the common pathway obtained from the co-expression network approach and the DAVID method. However, the pathway quantity obtained from co-expression network approach was increased compared with the DAVID method; A total of 447 differential pathways were selected using co-expression network method, while only 2 significant pathways were identified using the traditional DAVID). Therefore, the present study demonstrated that the co-expression network method exhibited increased efficiency compared with DAVID (data not shown).

\section{Discussion}

In the present study, a novel method of identifying pathway-based biomarkers in HCM was developed, by investigating dynamic interactions between molecules associated with pathogenesis through a co-expression network strategy. The results of the present study identified 447 differential pathways between HCM and normal samples, including folding of actin by CCT/TRiC, purine ribonucleoside monophosphate biosynthesis, and cooperation of prefoldin and $\mathrm{TRiC} / \mathrm{CCT}$ in actin and tubulin folding.

Actin, as a ubiquitous protein, serves functions in numerous cellular processes, including the maintenance of cell motility, cell shape, mitosis and intracellular transport processes $(29,30)$. Notably, the conserved nature of the amino acid sequence of actin indicates that mutated residues may affect basic functions, including actomyosin interactions and actin-actin interactions involved in polymerization (31). In previous studies, seven recognized actin mutations have been demonstrated to be associated with HCM $(30,32)$. Alterations may be induced by environmental factors, including diet, exercise, or by the cellular protein folding machinery. Previous studies have suggested that chaperone complexes (TRiC and CCT) may assist the folding of actin $(33,34)$. Vang et al $(35)$ demonstrated that protein-folding pathways serve a role in disease progression for actin mutations associated with HCM. Consistent with previous studies, the differentially expressed pathway of folding of actin by CCT/TRiC was identified in the present study. Therefore, it may be inferred that the disturbance of actin folding may be 
the molecular basis for the subsequent initiation of the hypertrophic pathway, resulting in the occurrence of HCM.

An additional altered pathway, purine ribonucleoside monophosphate biosynthesis, was screened out in the present study. As demonstrated in a recent study, energy flow is generated from ribose in purine ribonucleoside monophosphates (36). The inability to maintain normal ATP utilization may be the primary abnormality in $\operatorname{HCM}(37,38)$. Therefore, the differential pathway of purine ribonucleoside monophosphate biosynthesis may be important in the progression of HCM, which may involve a response to the disruption of energy homeostasis.

In conclusion, based on a co-expression network, differential pathways, including folding of actin by CCT/TRiC and purine ribonucleoside monophosphate biosynthesis, were successfully identified and these pathways may be involved in the pathogenic process of HCM. The results of the present study may be applied clinically for the diagnosis, prognostic management and treatment of patients with HCM.

\section{References}

1. McLeod CJ, Bos JM, Theis JL, Edwards WD, Gersh BJ, Ommen SR and Ackerman MJ: Histologic characterization of hypertrophic cardiomyopathy with and without myofilament mutations. Am Heart J 158: 799-805, 2009.

2. Ashrafian H and Watkins H: Reviews of translational medicine and genomics in cardiovascular disease: New disease taxonomy and therapeutic implications cardiomyopathies: Therapeutics based on molecular phenotype. J Am Coll Cardiol 49: 1251-1264, 2007.

3. Watkins H, McKenna WJ, Thierfelder L, Suk HJ, Anan R, O'Donoghue A, Spirito P, Matsumori A, Moravec CS, Seidman JG, et al: Mutations in the genes for cardiac troponin T and alpha-tropomyosin in hypertrophic cardiomyopathy. N Engl J Med 332: 1058-1065, 1995.

4. Maron BJ, Doerer JJ, Haas TS, Tierney DM and Mueller FO: Sudden deaths in young competitive athletes: Analysis of 1866 deaths in the United States, 1980-2006. Circulation 119: 1085-1092, 2009.

5. Bradley EW, Ruan MM, Vrable A and Oursler MJ: Pathway crosstalk between Ras/Raf and PI3K in promotion of M-CSF-induced MEK/ERK-mediated osteoclast survival. J Cell Biochem 104: 1439-1451, 2008.

6. Efthimiadis GK, Pagourelias ED, Gossios T and Zegkos T: Hypertrophic cardiomyopathy in 2013: Current speculations and future perspectives. World J Cardiol 6: 26, 2014.

7. Roma-Rodrigues $\mathrm{C}$ and Fernandes AR: Genetics of hypertrophic cardiomyopathy: Advances and pitfalls in molecular diagnosis and therapy. Appl Clin Genet 7: 195, 2014.

8. Liang D, Han G, Feng X, Sun J, Duan Y and Lei H: Concerted perturbation observed in a hub network in Alzheimer's disease. PLoS One 7: e40498, 2012.

9. Green M and Karp P: The outcomes of pathway database computations depend on pathway ontology. Nucleic Acids Res 34: 3687-3697, 2006.

10. Glazko GV and Emmertstreib F: Unite and conquer: Univariate and multivariate approaches for finding differentially expressed gene sets. Bioinformatics 25: 2348-2354, 2009.

11. Zhai DK, Liu B, Bai XF and Wen JA: Identification of biomarkers and pathway-related modules involved in ovarian cancer based on topological centralities. J BUON 21: 208-220, 2016.

12. Khatri P, Sirota M and Butte AJ: Ten years of pathway analysis: Current approaches and outstanding challenges. PLoS Comput Biol 8: e1002375, 2012

13. Kanehisa M and Goto S: KEGG: Kyoto encyclopedia of genes and genomes. Nucleic Acids Res 28: 27-30, 2000.

14. Liu X, Tang WH, Zhao XM and Chen L: A network approach to predict pathogenic genes for Fusarium graminearum. PLoS One 5: pii: e13021, 2010.

15. Chen L, Wang RS and Zhang XS (eds): Biomolecular Networks: Methods and Applications in Systems Biology. Wiley, 2009.

16. Bandyopadhyay S, Mehta M, Kuo D, Sung MK, Chuang R, Jaehnig EJ, Bodenmiller B, Licon K, Copeland W, Shales M, et al: Rewiring of genetic networks in response to DNA damage. Science 330: 1385-1389, 2010
17. Irizarry RA, Hobbs B, Collin F, Beazer-Barclay YD, Antonellis KJ Scherf U and Speed TP: Exploration, normalization, and summaries of high density oligonucleotide array probe level data. Biostatistics 4: 249-264, 2003.

18. Bolstad BM,Irizarry RA,Astrand M and Speed TP: A comparison of normalization methods for high density oligonucleotide array data based on variance and bias. Bioinformatics 19: 185-193, 2003.

19. Pepper SD, Saunders EK, Edwards LE, Wilson CL and Miller CJ: The utility of MAS5 expression summary and detection call algorithms. BMC Bioinformatics 8: 273, 2007.

20. Croft D: Building models using Reactome pathways as templates. In: In Silico Systems Biology Springer 1021: pp273-283, 2013.

21. Dawson JA, Ye S and Kendziorski C: R/EBcoexpress: An empirical Bayesian framework for discovering differential co-expression. Bioinformatics 28: 1939-1940, 2012.

22. Manfield IW, Jen CH, Pinney JW, Michalopoulos I, Bradford JR, Gilmartin PM and Westhead DR: Arabidopsis Co-expression Tool (ACT): Web server tools for microarray-based gene expression analysis. Nucleic Acids Res 34 (Web Server Issue): W504-W509, 2006.

23. Langfelder P and Horvath S: WGCNA: An R package for weighted correlation network analysis. BMC Bioinformatics 9: 559,2008

24. Dawson JA and Kendziorski C: An empirical bayesian approach for identifying differential coexpression in high-throughput experiments. Biometrics 68: 455-465, 2012.

25. Fraley $\mathrm{C}$ and Raftery AE: Model-based clustering, discriminant analysis and density estimation. J Am Stat Assoc 97: 611-631, 2002.

26. Dawson JA, Dawson M and Ebarrays D: Package 'EBcoexpress'.

27. Huang da W, Sherman BT and Lempicki RA: Systematic and integrative analysis of large gene lists using DAVID bioinformatics resources. Nat Protoc 4: 44-57, 2009.

28. Ford G, Xu Z, Gates A, Jiang J and Ford BD: Expression analysis systematic explorer (EASE) analysis reveals differential gene expression in permanent and transient focal stroke rat models. Brain Res 1071: 226-236, 2006.

29. Huxley HE: Fifty years of muscle and the sliding filament hypothesis. Eur J Biochem 271: 1403-1415, 2004.

30. Kodama A, Lechler T and Fuchs E: Coordinating cytoskeletal tracks to polarize cellular movements. J Cell Biol 167: 203-207, 2004.

31. Bookwalter CS and Trybus KM: Functional consequences of a mutation in an expressed human alpha-cardiac actin at a site implicated in familial hypertrophic cardiomyopathy. J Biol Chem 281: 16777-16784, 2006.

32. Van Driest SL, Ellsworth EG, Ommen SR, Tajik AJ, Gersh BJ and Ackerman MJ: Prevalence and spectrum of thin filament mutations in an outpatient referral population with hypertrophic cardiomyopathy. Circulation 108: 445-451, 2003.

33. Rommelaere H, Waterschoot D, Neirynck K, Vandekerckhove J and Ampe C: Structural plasticity of functional actin: Pictures of actin binding protein and polymer interfaces. Structure 11: 1279-1289, 2003 .

34. Hansen WJ, Cowan NJ and Welch WJ: Prefoldin-nascent chain complexes in the folding of cytoskeletal proteins. J Cell Biol 145: 265-277, 1999.

35. Vang S, Corydon TJ, Børglum AD, Scott MD, Frydman J, Mogensen J, Gregersen N and Bross P: Actin mutations in hypertrophic and dilated cardiomyopathy cause inefficient protein folding and perturbed filament formation. FEBS J 272: 2037-2049, 2005

36. Hibbs JB Jr, Vavrin Z and Cox JE: Complex coordinated extracellular metabolism: Acid phosphatases activate diluted human leukocyte proteins to generate energy flow as NADPH from purine nucleotide ribose. Redox Biol 8: 271-284, 2016.

37. Spindler M, Saupe K, Christe M, Sweeney H, Seidman C, Seidman JG and Ingwall JS: Diastolic dysfunction and altered energetics in the alphaMHC403/+ mouse model of familial hypertrophic cardiomyopathy. J Clin Invest 101: 1775-1783, 1998.

38. Blair E, Redwood C, Ashrafian H, Oliveira M, Broxholme J, Kerr B, Salmon A, Ostman-Smith I and Watkins H: Mutations in the gamma(2) subunit of AMP-activated protein kinase cause familial hypertrophic cardiomyopathy: Evidence for the central role of energy compromise in disease pathogenesis. Hum Mol Genet 10: 1215-1220, 2001. 